Supplementary Materials for

\title{
Suppressing Subcapsular Sinus Macrophages Enhances Transport of Nanovaccines to Lymph Node Follicles for Robust Humoral Immunity
}

Yi-Nan Zhang ${ }^{1,2}$, Wilson Poon ${ }^{1,2}$, Elana Sefton ${ }^{1,2}$, Warren C.W. Chan*1,2,3,4,5

${ }^{1}$ Institute of Biomaterials \& Biomedical Engineering, University of Toronto, Toronto, ON M5S 3G9, Canada

${ }^{2}$ Terrence Donnelly Centre for Cellular \& Biomolecular Research, University of Toronto, Toronto, ON M5S 3E1, Canada

${ }^{3}$ Department of Chemical Engineering \& Applied Chemistry, University of Toronto, Toronto, ON M5S 3E5, Canada

${ }^{4}$ Department of Materials Science \& Engineering, University of Toronto, Toronto, ON M5S

1A1, Canada

${ }^{5}$ Department of Chemistry, University of Toronto, Toronto, ON, M5S 3H6, Canada

${ }^{*}$ Corresponding author: warren.chan@utoronto.ca. 
Supplementary Figures and Table:

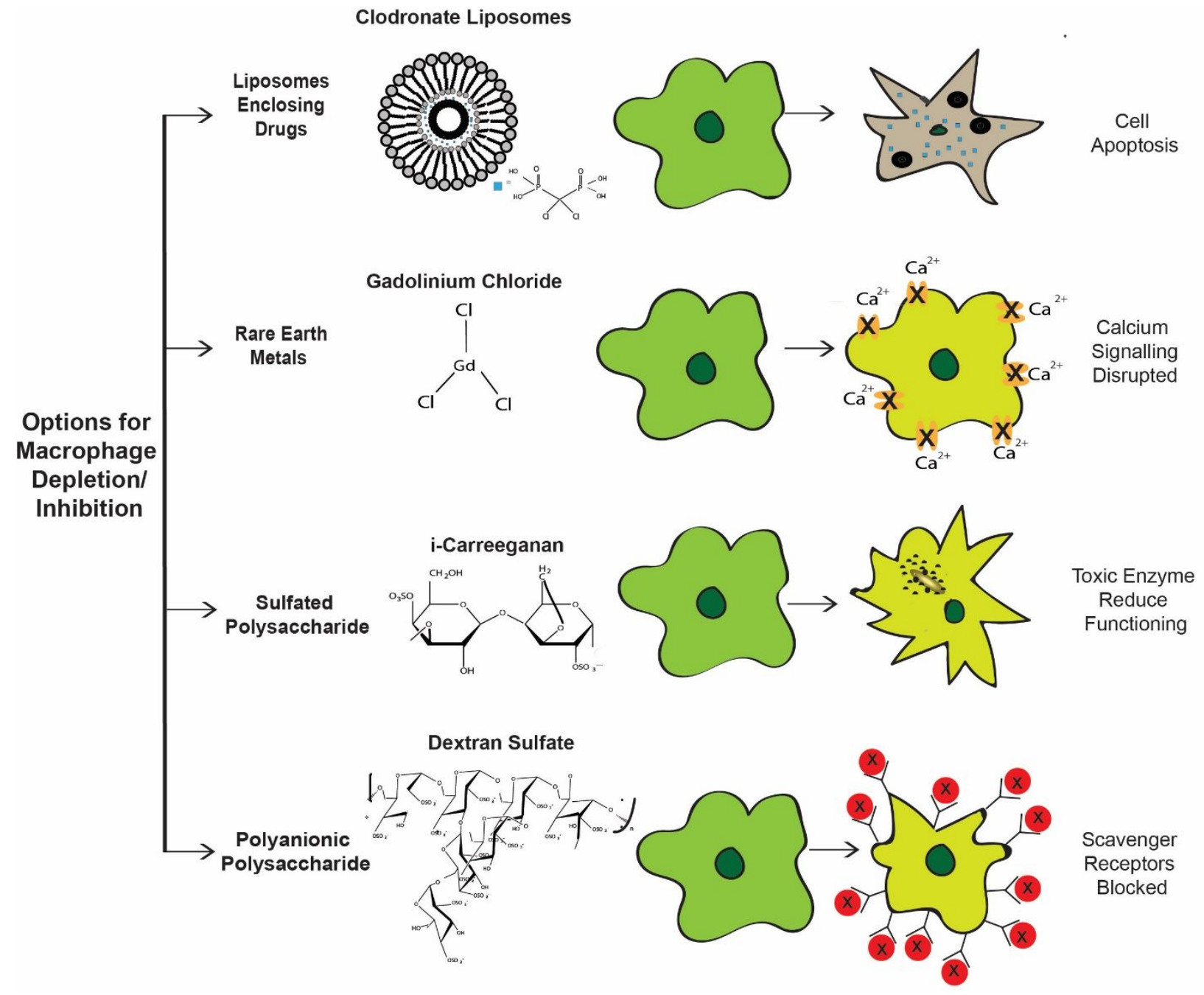

Fig. S1. Mechanism of macrophage inhibitors. Clodronate liposomes were used to deplete SCS macrophages in lymph nodes. Other macrophage inhibitors including gadolinium chloride $\left(\mathrm{GdCl}_{3}\right)$, carrageenan (CGN), or dextran sulfate 500 (DS500) were used to inhibit SCS macrophage uptake function 


\section{A Measured from histology (Prox1)}

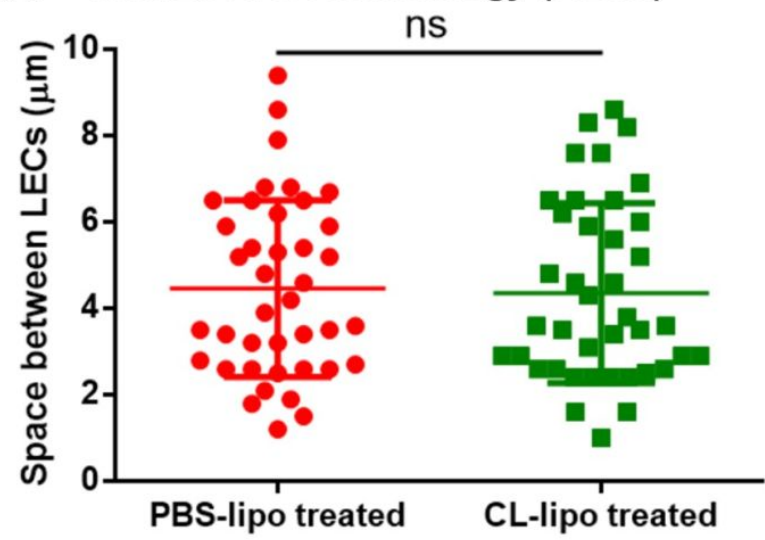

B Measured from TEM

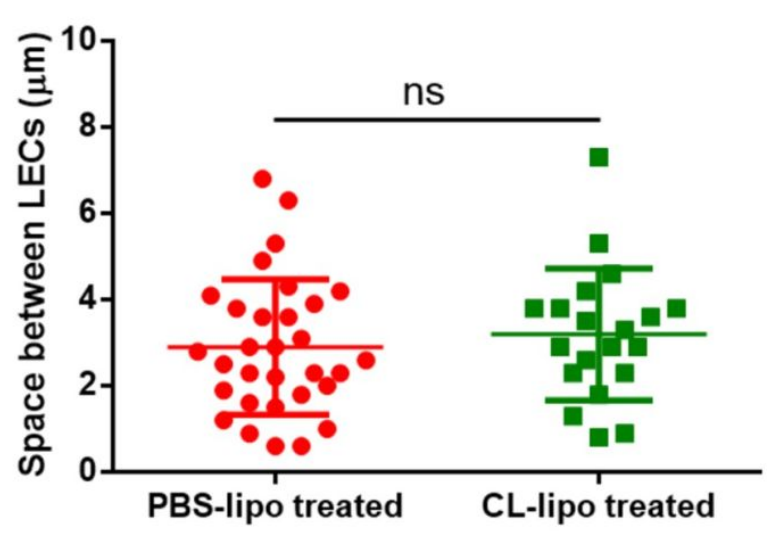

Fig. S2. Quantification of space between lymphatic endothelial cells (LECs). (A) Spaces between LECs were measured based on Prox1 antibody stain $(n=40)$. (B) Spaces between LECs were measured based on TEM $(n>20)$. Spaces between LECs was measured after PBS liposome and clodronate liposome treatment. PBS-lipo represents PBS liposome and CL-lipo represents clodronate liposome in the figure. Data shown as mean $\pm \mathrm{SD}$. The statistical analysis was studied by an unpaired $t$ test. 


\section{A PBS-lipo treated B CL-lipo treated}
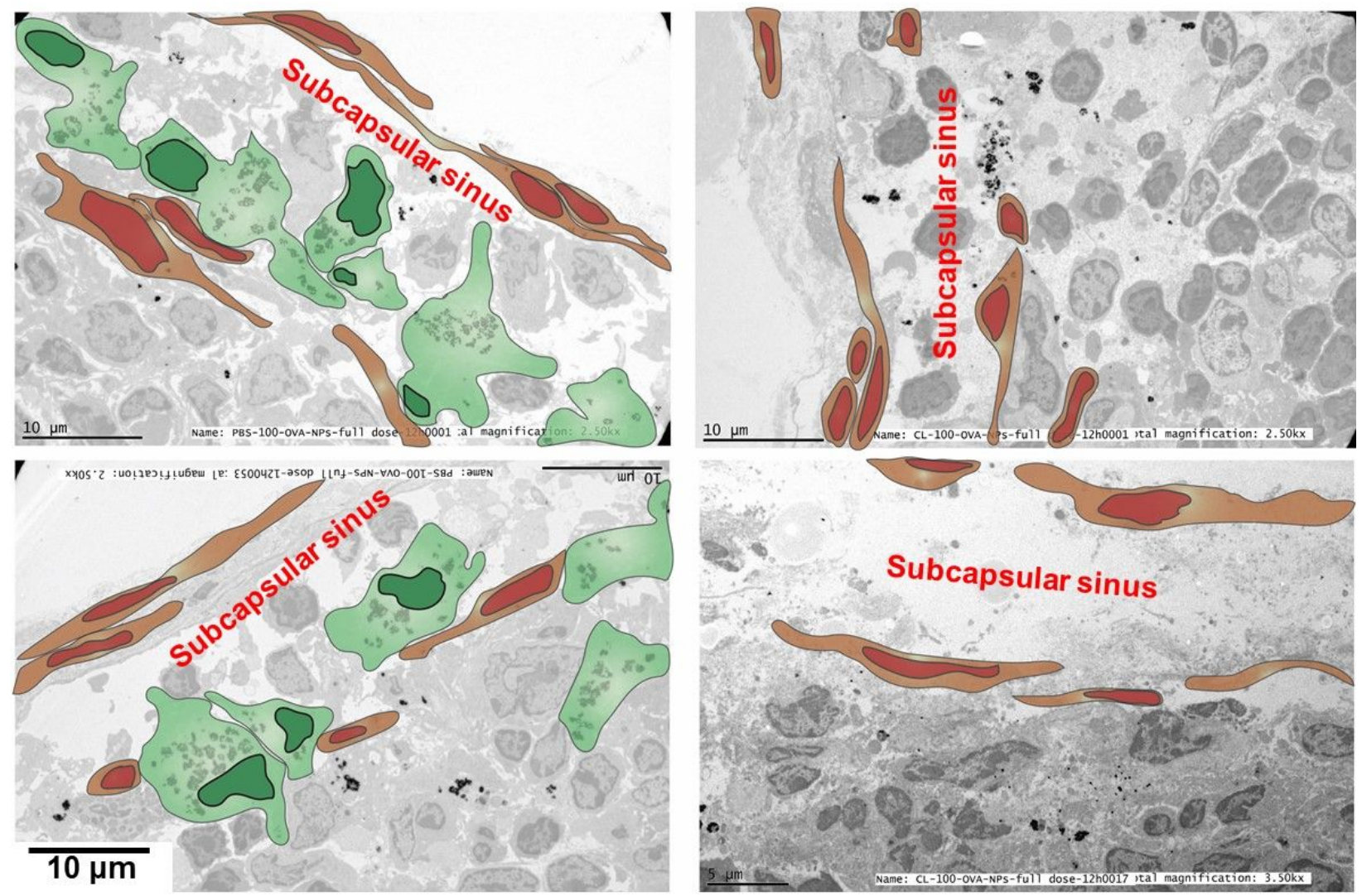

Fig. S3. Representative TEM images of lymph node subcapsular sinus after intradermal footpad injection of $100 \mathrm{~nm}$ OVA-AuNP nanovaccines at 12 hours. The mice were pretreated with (A) PBS liposome or (B) clodronate liposomes before administration of nanovaccine. Green colors represent SCS macrophages, brown colors represent LECs. Black colors are the nanovaccines. 

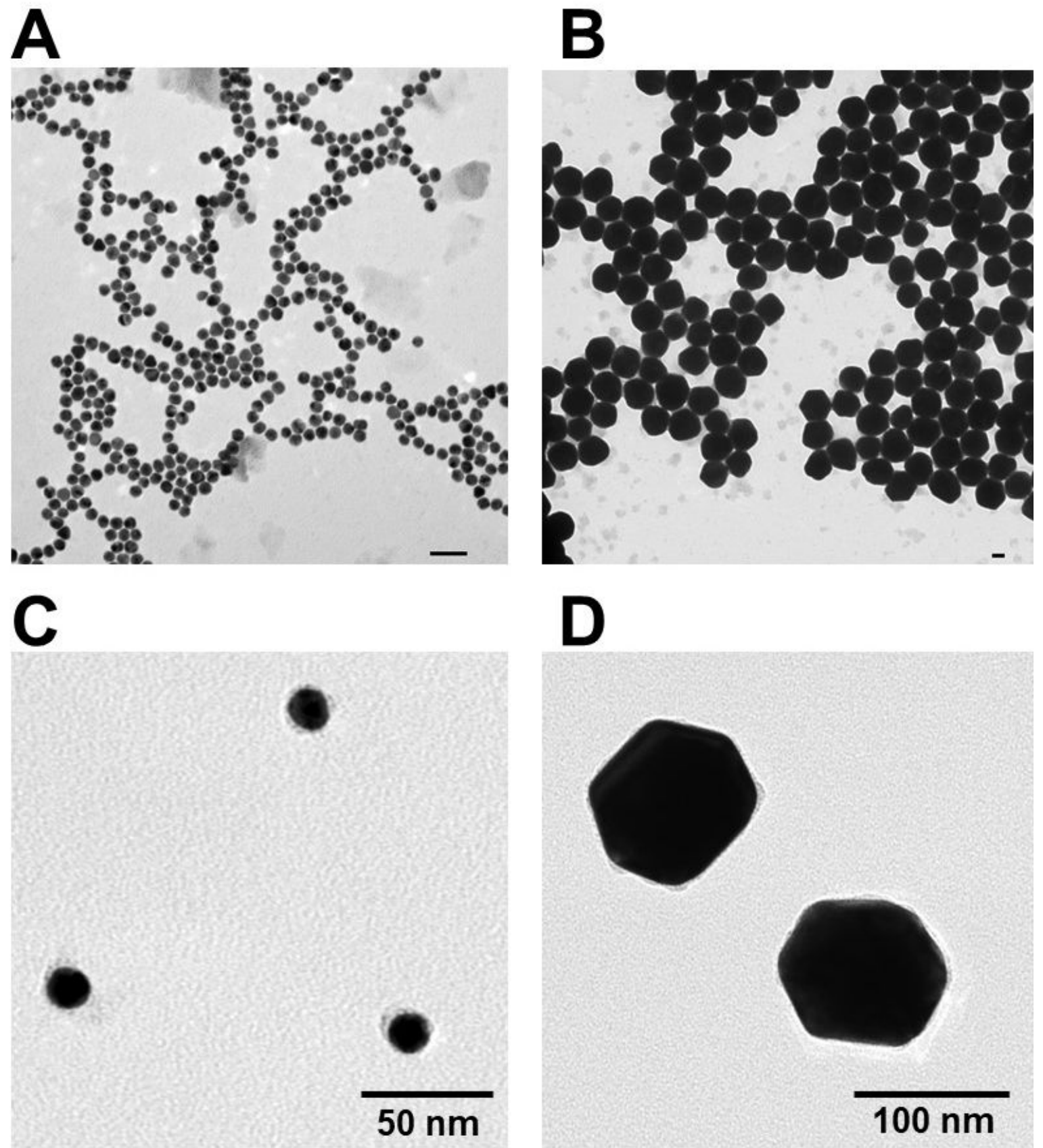

Fig. S4. Characterization of nanoparticles and nanovaccines. TEM images of (A) 15 and (B) $100 \mathrm{~nm}$ citrate-coated gold nanoparticles (citrate-AuNPs). Scale $=50 \mathrm{~nm}$. TEM images of ovalbumin-coated gold nanoparticles (OVA-AuNPs) with core sizes of (C) 15 and (D) $100 \mathrm{~nm}$. 
Table. S1. Physiochemical properties of nanoparticles and nanovaccines

\begin{tabular}{|c|c|c|c|c|c|}
\hline \multicolumn{2}{|c|}{ Nanoparticle } & \multicolumn{3}{|c|}{ Physicochemical Properties } \\
\hline Size & Surface Ligands & $\begin{array}{c}\text { Inorganic Diameter } \\
(\mathrm{nm})\end{array}$ & $\begin{array}{c}\text { Hydrodynamic } \\
\text { Diameter }(\mathrm{nm})\end{array}$ & $\begin{array}{c}\xi \text { Potential } \\
(\mathrm{mV})\end{array}$ & $\lambda_{\text {LSPR }}(\mathrm{nm})$ \\
\hline 15 & Citrate & $15.3 \pm 1.2$ & $19.2 \pm 0.5$ & $-9.7 \pm 1.6$ & 521 \\
& OVA & & $79.6 \pm 5.5$ & $-21.5 \pm 1.2$ & 528 \\
\hline 100 & Citrate & $109.0 \pm 5.9$ & $107.1 \pm 2.9$ & $-24.8 \pm 1.1$ & 575 \\
& OVA & & $165.7 \pm 6.5$ & $-22.5 \pm 1.3$ & 587 \\
\hline
\end{tabular}

\section{No. of OVA per AuNP}

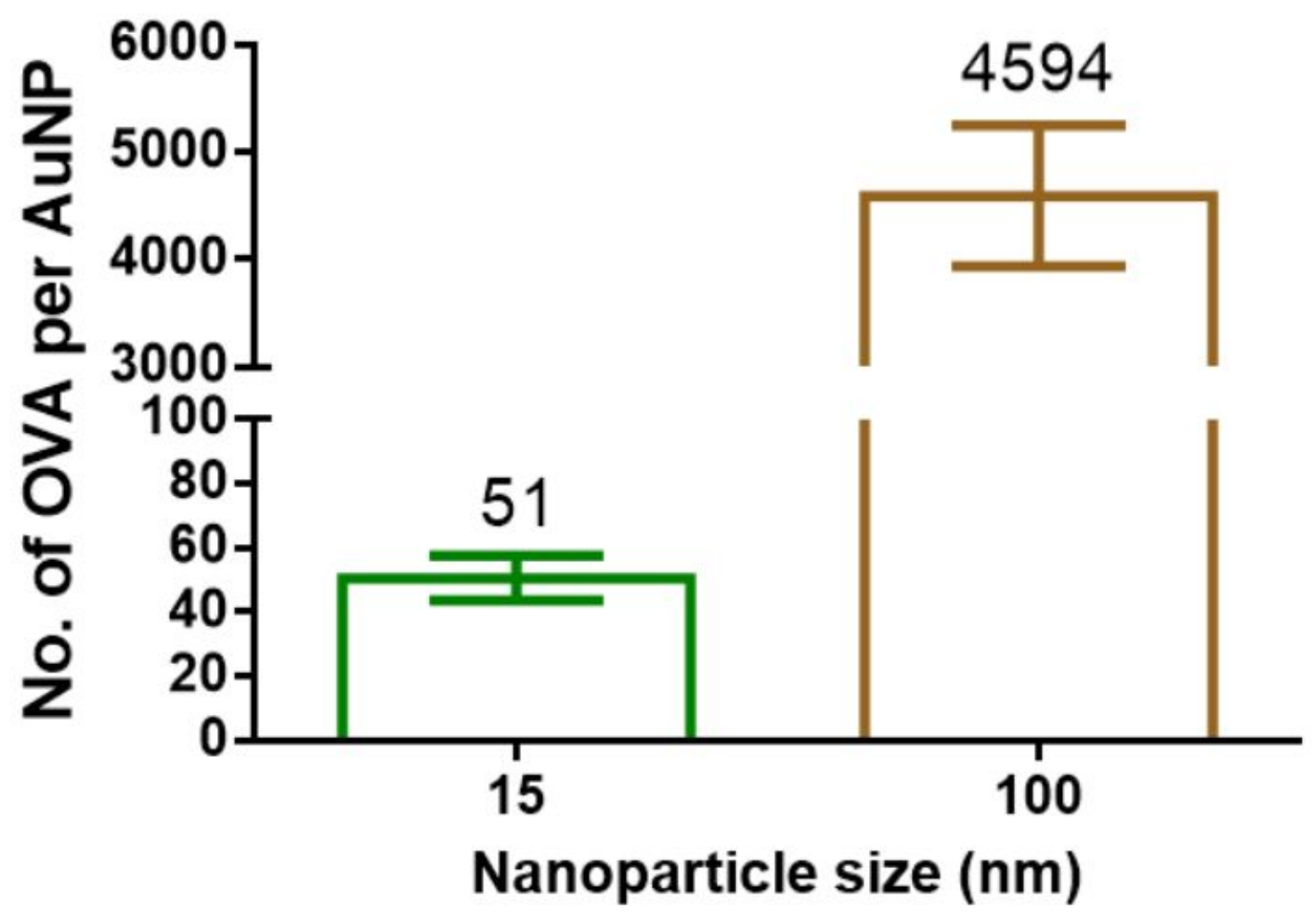

Fig. S5. Quantification of OVA protein amount on AuNPs using bicinchoninic acid (BCA) assay. The OVA-AuNP injection dose is normalized based on the same total amount of OVA antigen. 


\section{A PBS-lipo treated}
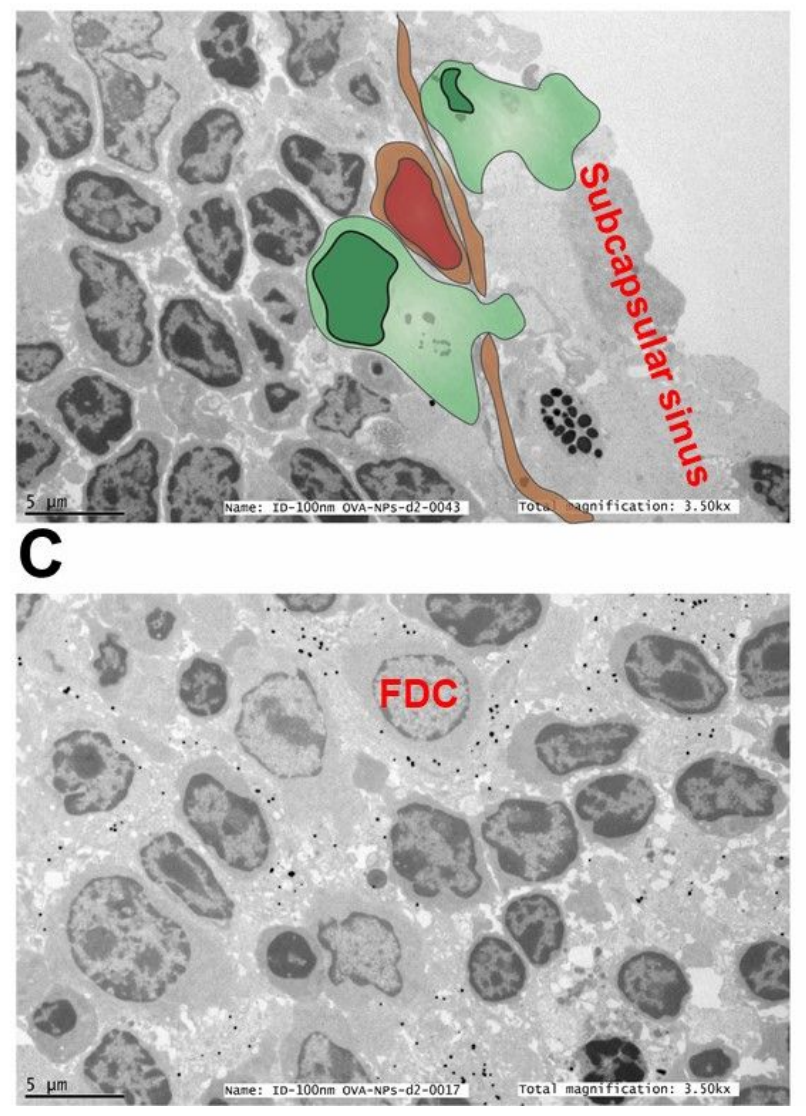

\section{B CL-lipo treated}
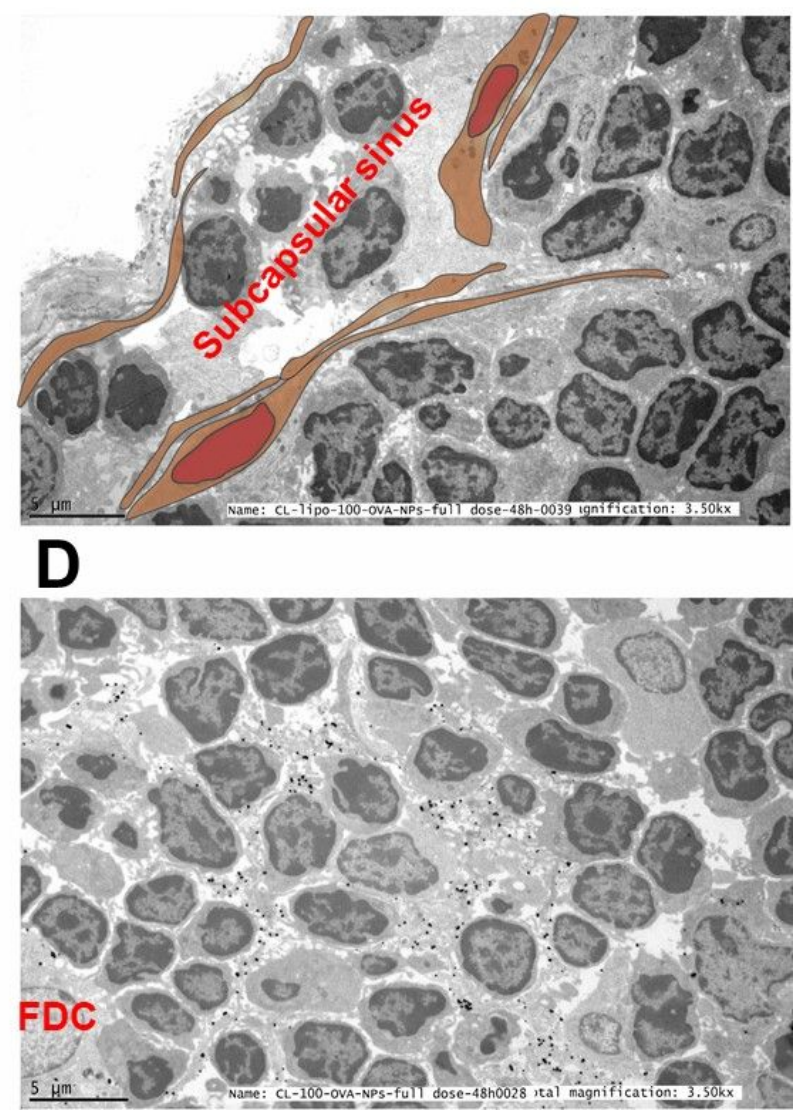

Fig. S6. Representative TEM images of lymph node after intradermal footpad injection of $100 \mathrm{~nm}$ OVA-AuNP nanovaccins at 48 hours. The mice were pretreated with PBS liposome or clodronate liposomes before administration of nanovaccine. TEM images of lymph node subcapsular sinus after (A) PBS liposome and (B) clodronate liposome treatments. TEM images of lymph node follicles after (C) PBS liposome and (D) clodronate liposome treatments. Nanovaccines are deposited on follicular dendritic cell dendrites. Green colors represent SCS macrophages, brown colors represent LECs. Black colors are the nanovaccines.

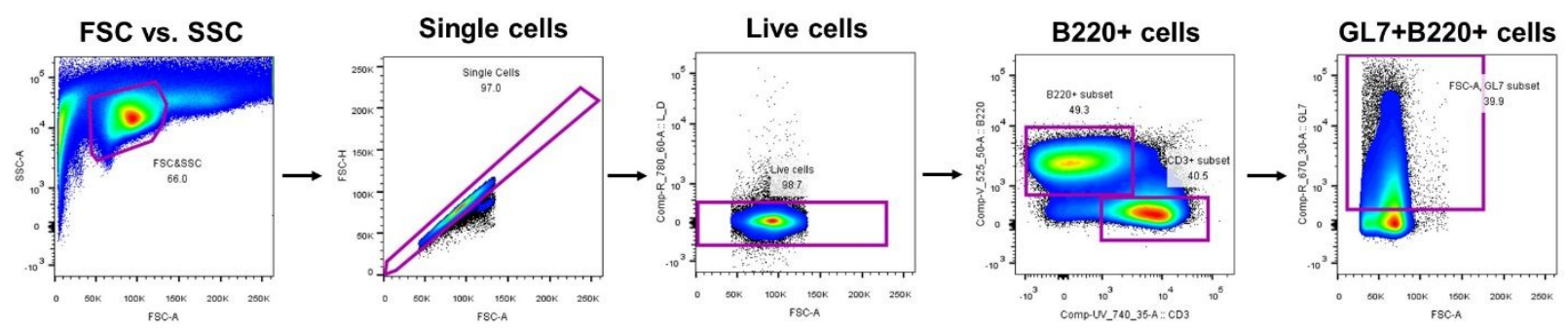

Fig. S7. Gating strategy for analyzing germinal center B cells after 5 weeks of immunization using $100 \mathrm{~nm}$ OVA-AuNPs. 


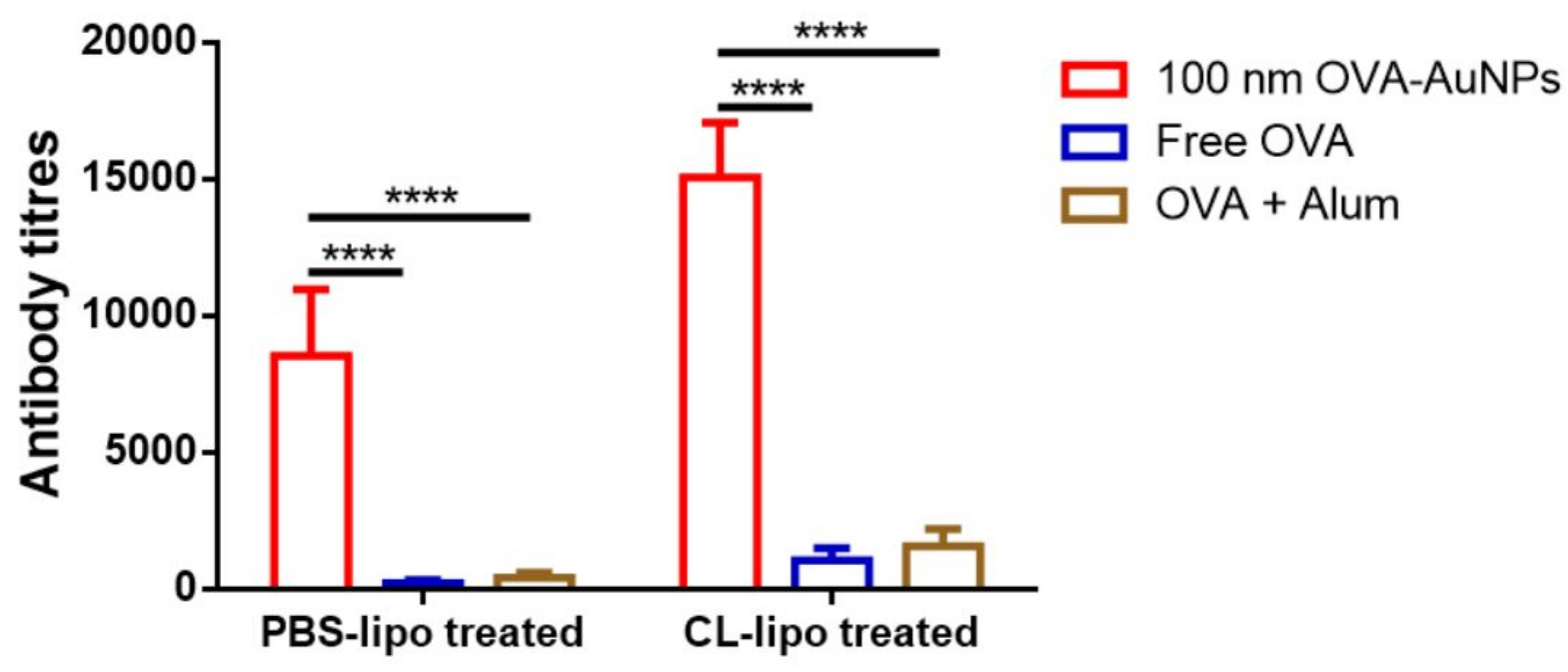

Fig. S8. Nanovaccines induce greater production of antigen specific antibodies compared to free antigen and antigen formulated with commercial adjuvant Alum in both PBS and Clodronate pretreated conditions $(\mathrm{n}=3-4$ mice/group). PBS-lipo represents PBS liposome and CL-lipo represents clodronate liposome in the figure. Data shown as mean $\pm \mathrm{SD} ; * * * \mathrm{P}<0.0001$. All $\mathrm{P}$ values are from two-way ANOVA followed by Tukey's multiple comparisons tests.
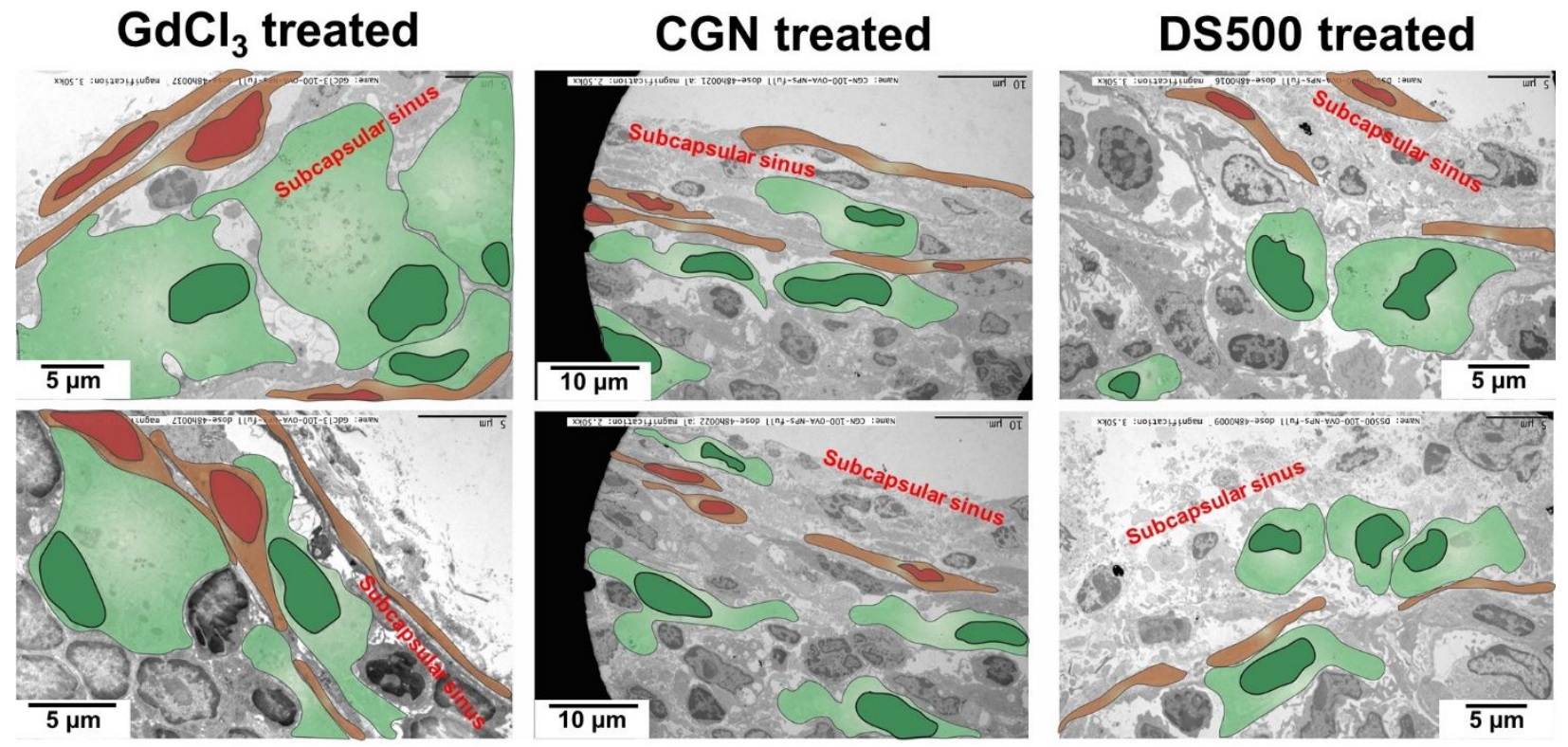

Fig. S9. Representative TEM images of lymph node after intradermal footpad injection of $100 \mathrm{~nm}$ OVA-AuNP nanovaccins at 48 hours. The mice were pretreated with $\mathrm{GdCl}_{3}$ or CGN or DS500 before administration of nanovaccines. Green colors represent SCS macrophages, brown colors represent LECs. 


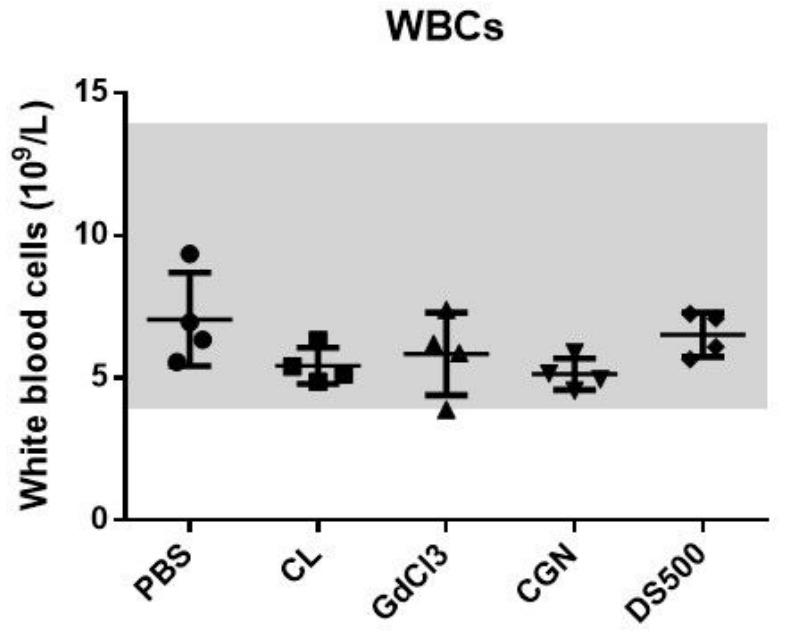

Monocytes

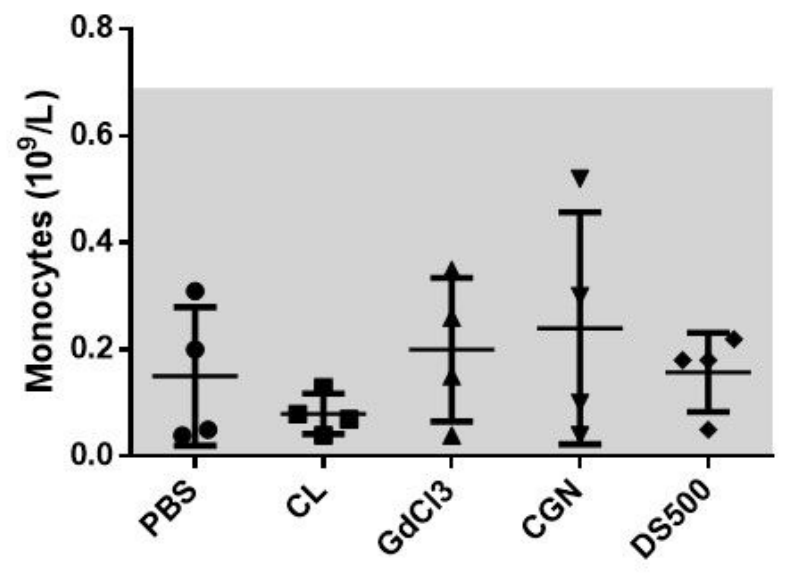

\section{Lymphocytes}

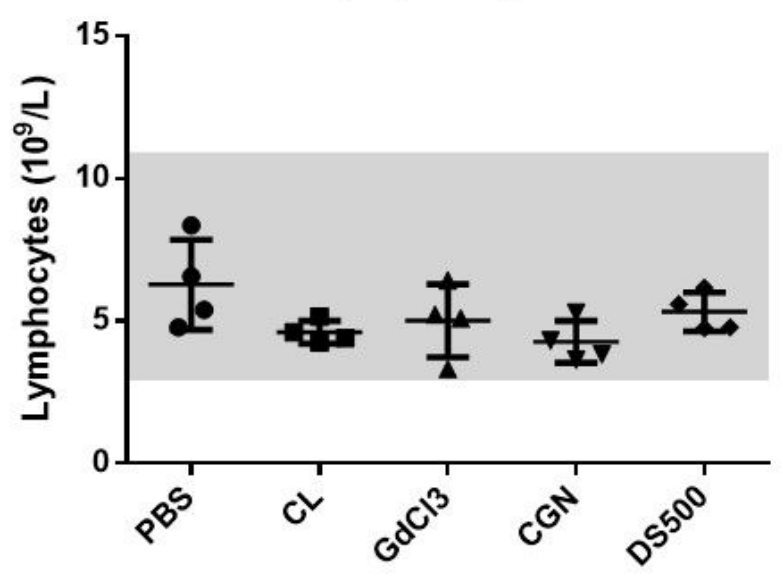

Neutrophils

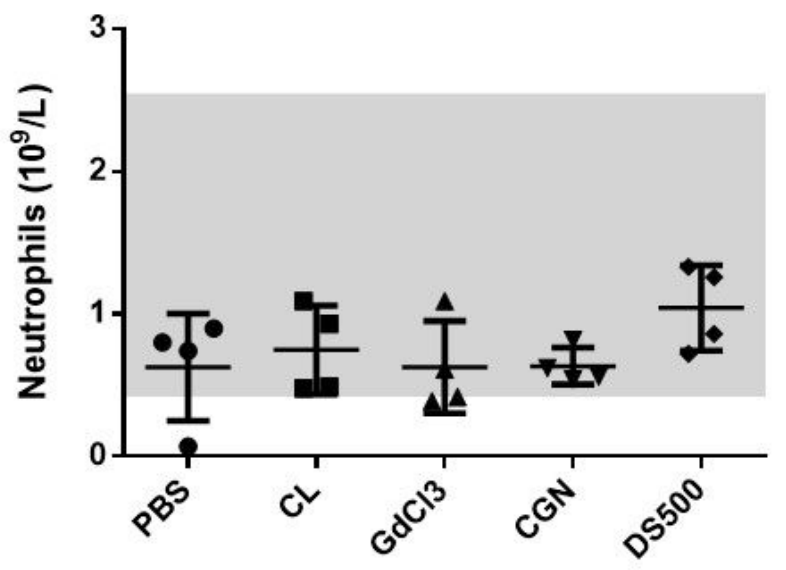

Fig. S10. Analysis of immune cells. Immune cell counts from blood of C57BL/6 mice 3 days after intradermal injection of macrophage inhibitors $(0.1 \mathrm{mg}$ per footpad, in total $0.4 \mathrm{mg}$ for four footpads) and PBS controls ( $\mathrm{n}=4$ mice/group). WBC represents white blood cells. Grey shaded area represents normal range of cell counts for $\mathrm{C} 57 \mathrm{BL} / 6$ mice. 
RBCs
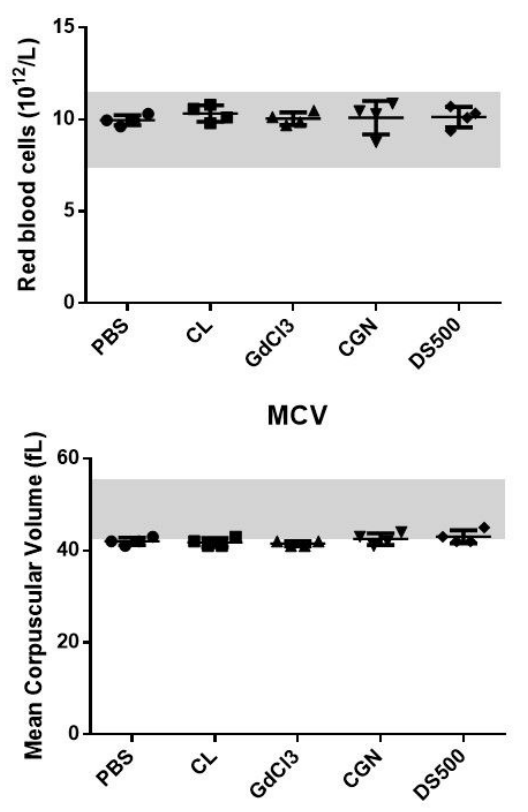

PLT

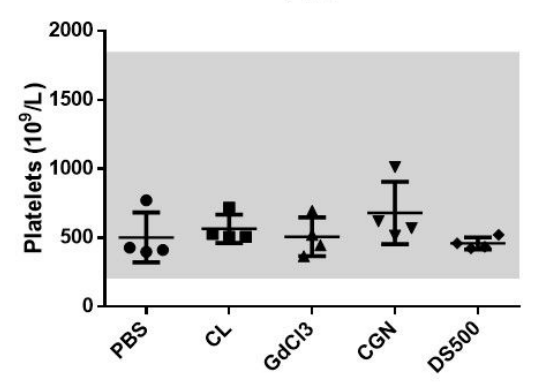

HGB
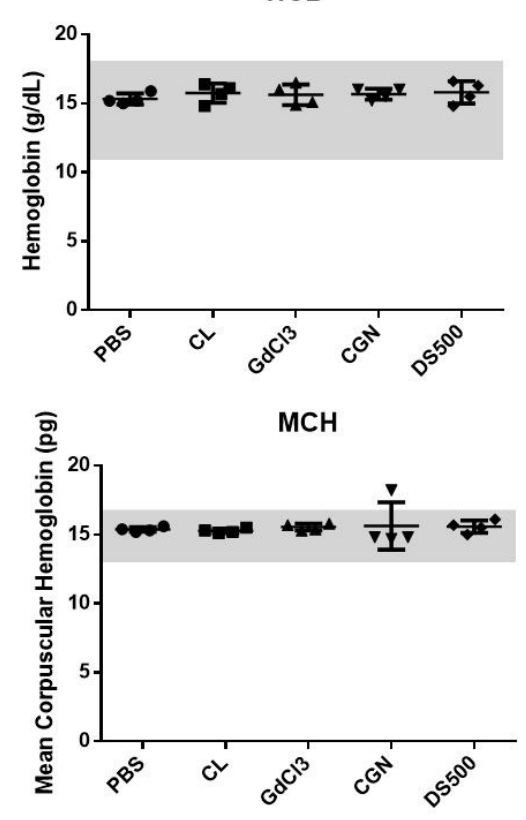

MPV

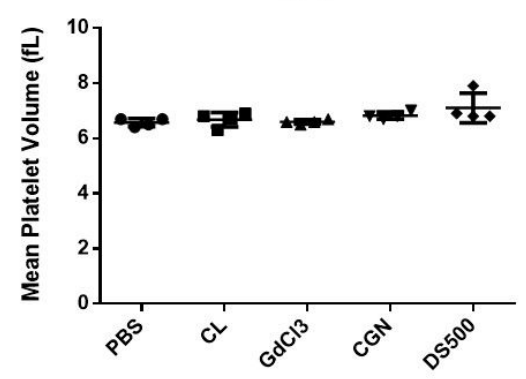

HCT
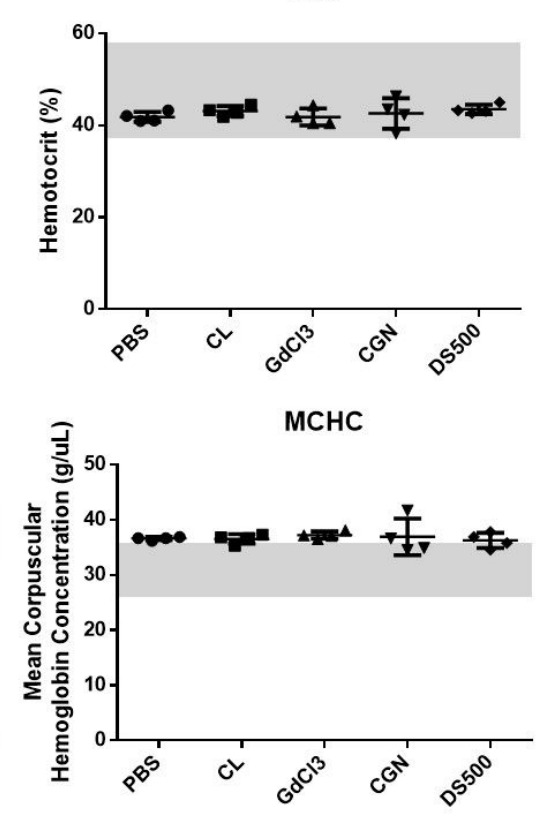

PCT

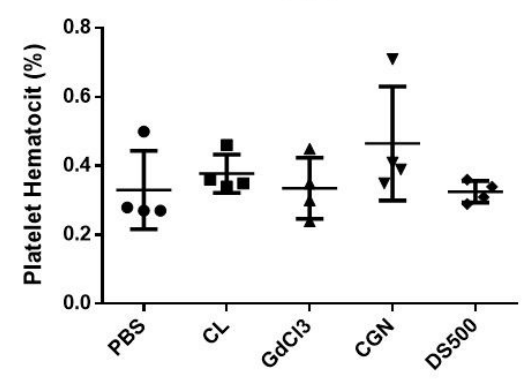

Fig. S11. Hematology study. Hematology analysis of C57BL/6 mice 3 days after intradermal injection of macrophage inhibitors $(0.1 \mathrm{mg}$ per footpad, in total $0.4 \mathrm{mg}$ for four footpads) and PBS controls ( $n=4$ mice/group). Parameters shown include red blood cell count (RBC), hemoglobin (HGB), hematocrit (HCT), mean corpuscular volume (MCV), mean corpuscular hemoglobin $(\mathrm{MCH})$, mean corpuscular hemoglobin concentration (MCHC), platelet count (PLT), mean platelet volume (MPV), and procalcitonin (PCT). Grey shaded area represents normal range for C57BL/6 mice. 
ALT

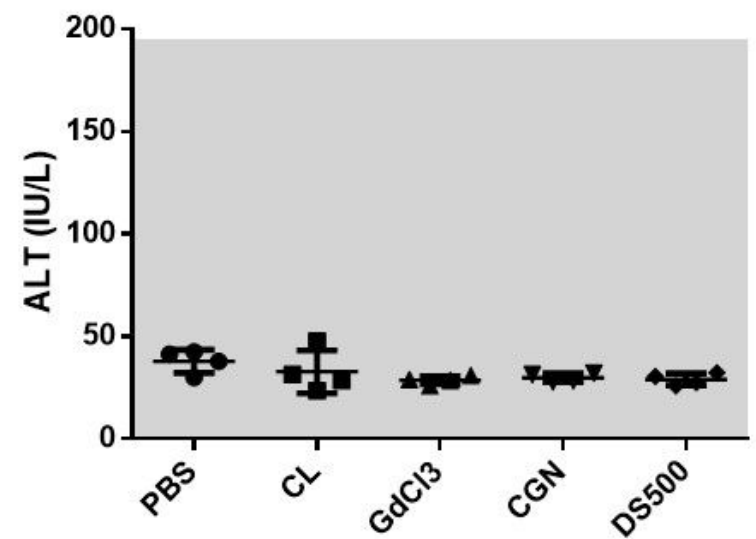

ALP

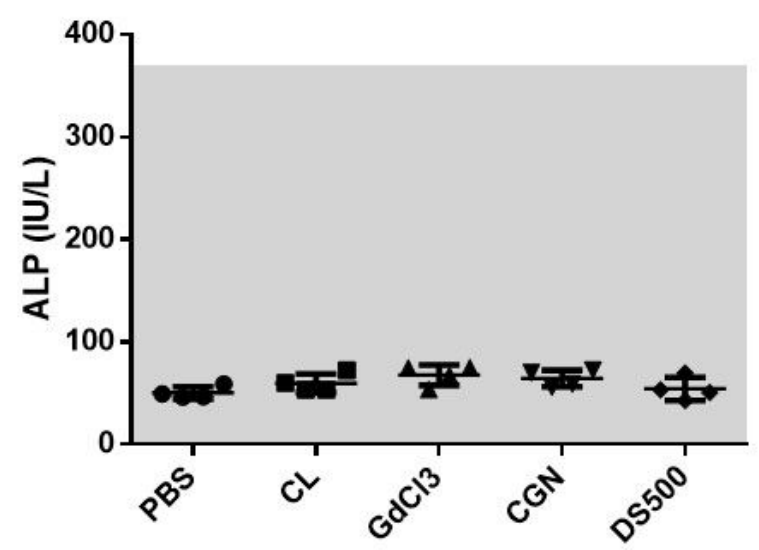

AST

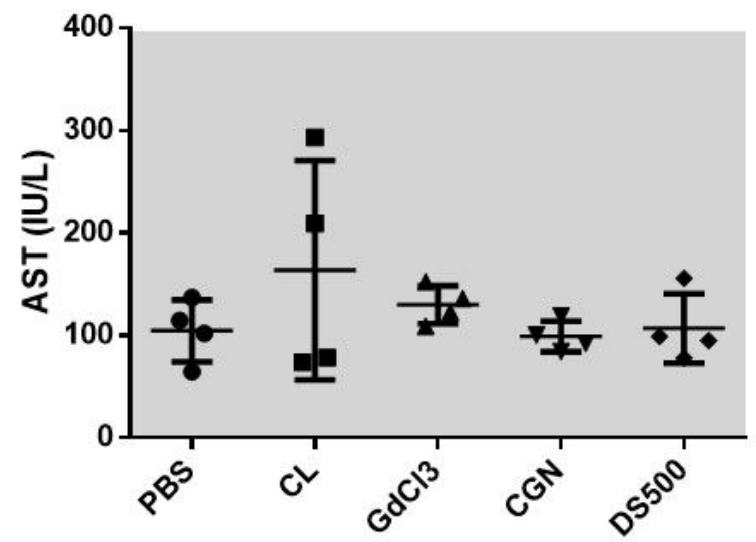

TBIL

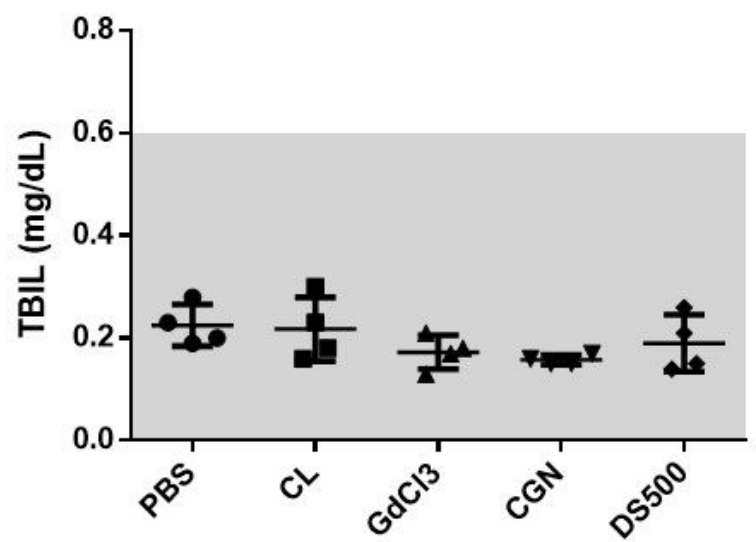

Fig. S12. Hepatotoxicity study. Hepatotoxicity analysis of serum markers alanine transaminase (ALT), aspartate aminotransferase (AST), alkaline phosphatase (ALP), and total bilirubin (TBIL) 3 days after intradermal injection of macrophage inhibitors $(0.1 \mathrm{mg}$ per footpad, in total $0.4 \mathrm{mg}$ for four footpads) and PBS controls ( $\mathrm{n}=4$ mice/group). Grey shaded area represents normal range for C57BL/6 mice. 

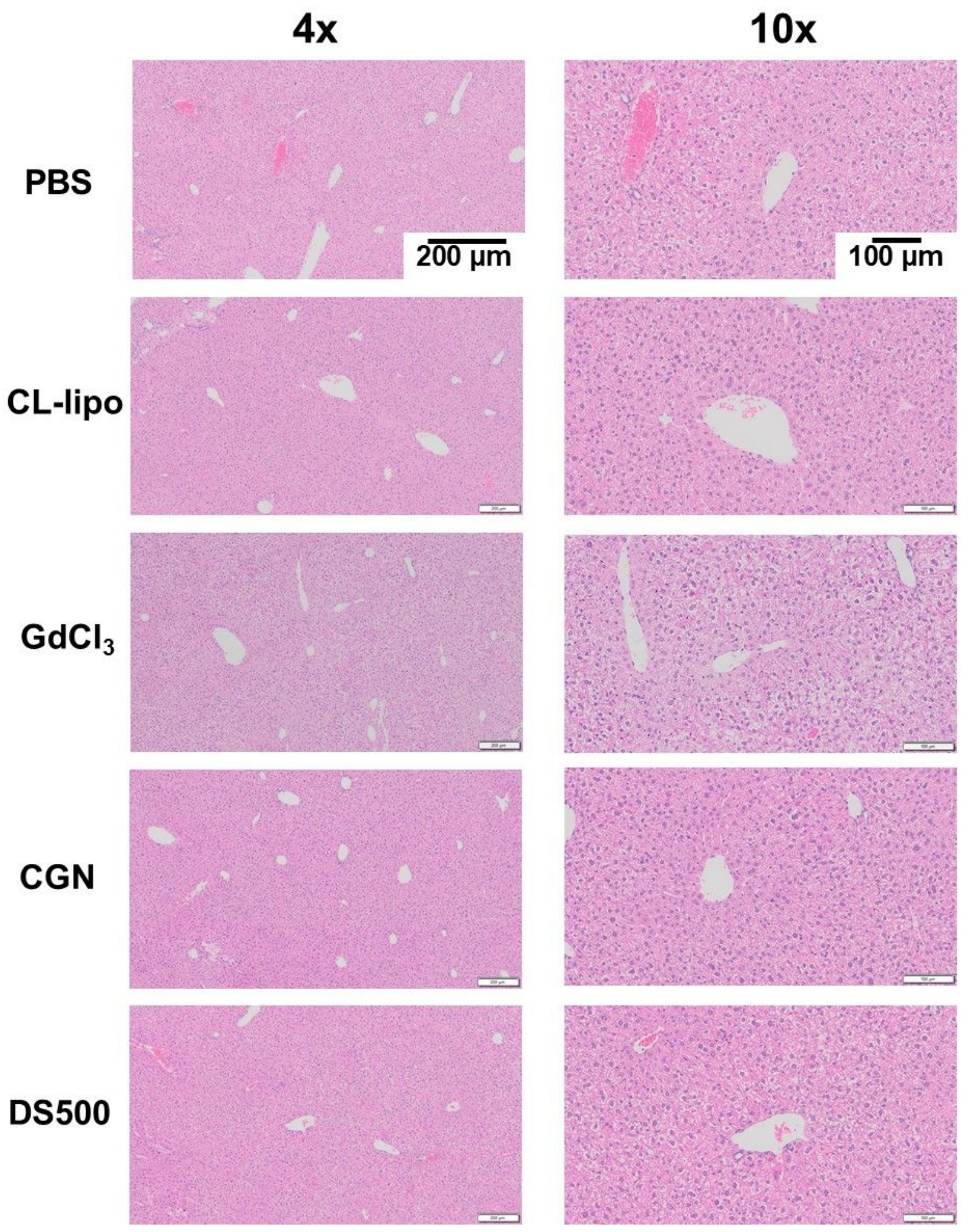

Fig. S13. Liver histology study. No inflammation was observed in the hepatic histological sections of C57BL/6 mice after treatment of PBS, clodronate liposomes, $\mathrm{GdCl}_{3}, \mathrm{CGN}$ and DS500 macrophage inhibitors $(0.1 \mathrm{mg}$ per footpad, in total $0.4 \mathrm{mg}$ for four footpads) at day 3 ( $\mathrm{n}=4$ mice/group). 
$4 \mathbf{x}$

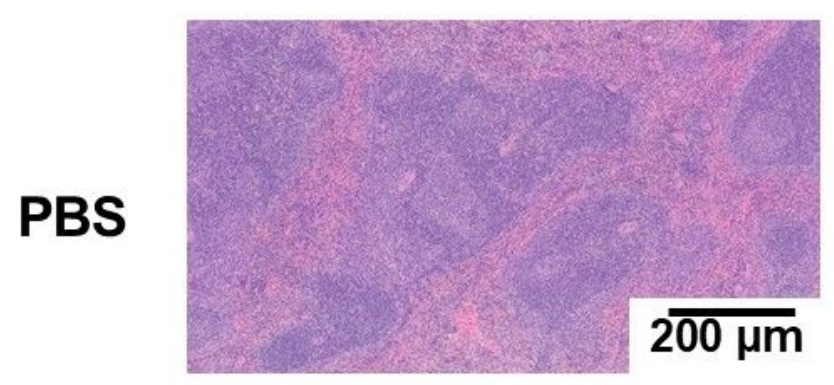

CL-lipo

$\mathrm{GdCl}_{3}$
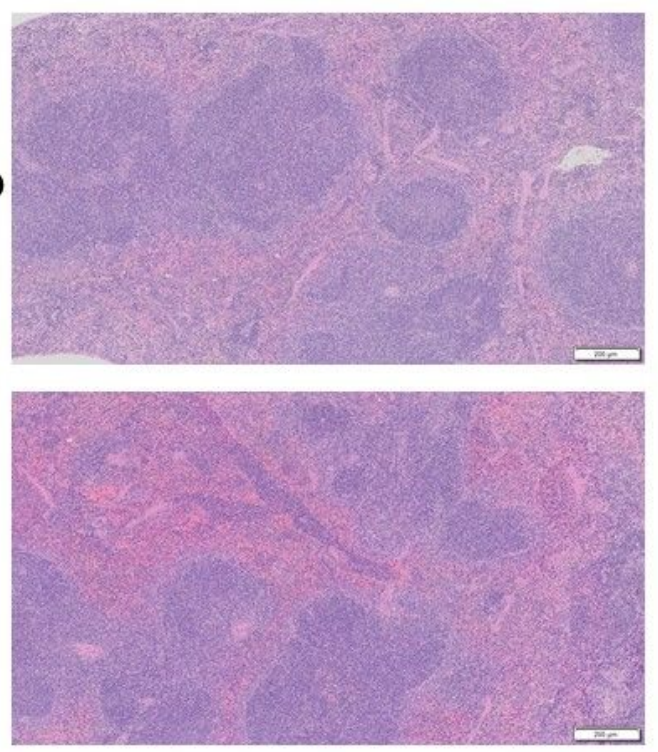

CGN

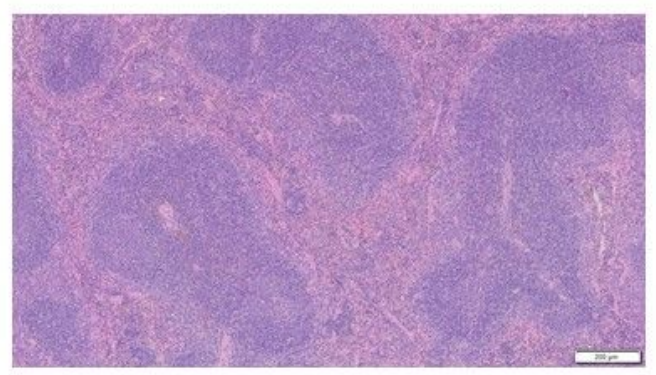

DS500

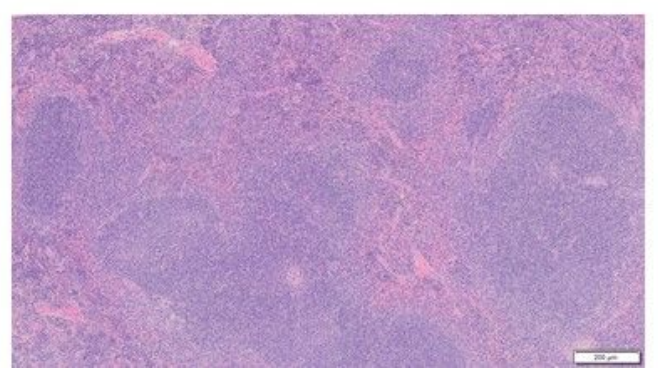

$10 x$
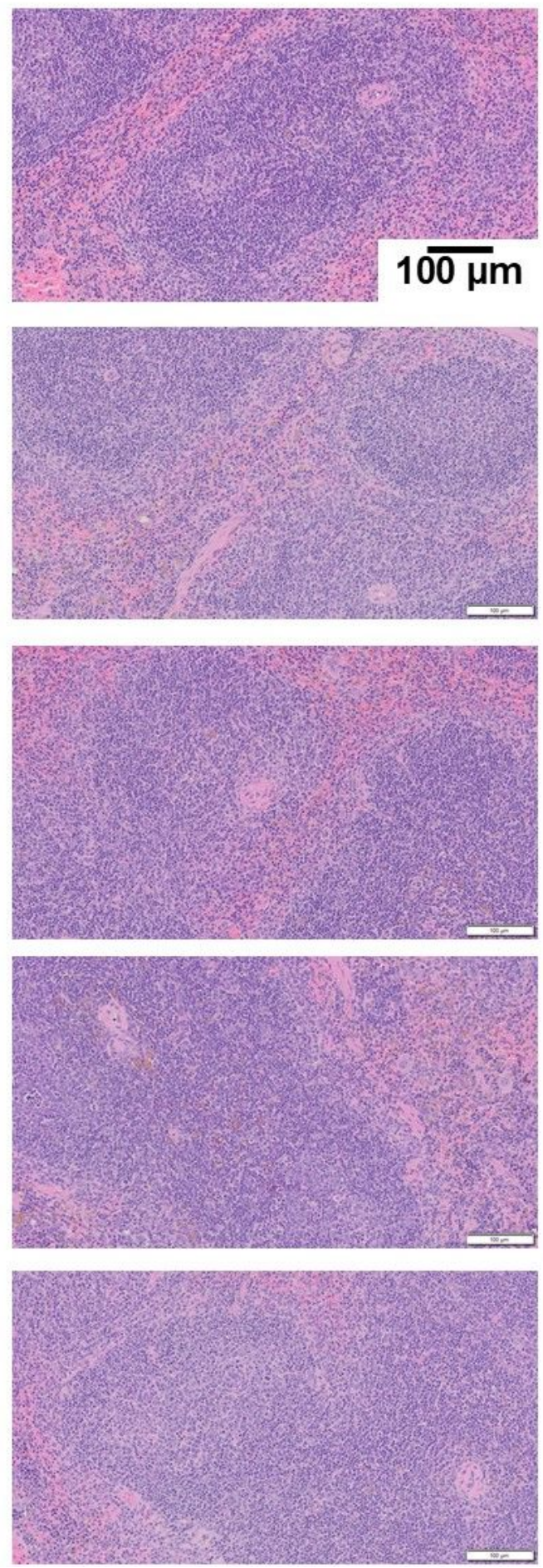

Fig. S14. Spleen pathology study. No inflammation was observed in the splenic histological sections of C57BL/6 mice after treatment of PBS, clodronate liposomes, $\mathrm{GdCl}_{3}, \mathrm{CGN}$ and DS500 macrophage inhibitors $(0.1 \mathrm{mg}$ per footpad, in total $0.4 \mathrm{mg}$ for four footpads) at day $3(\mathrm{n}=4$ mice/group). 

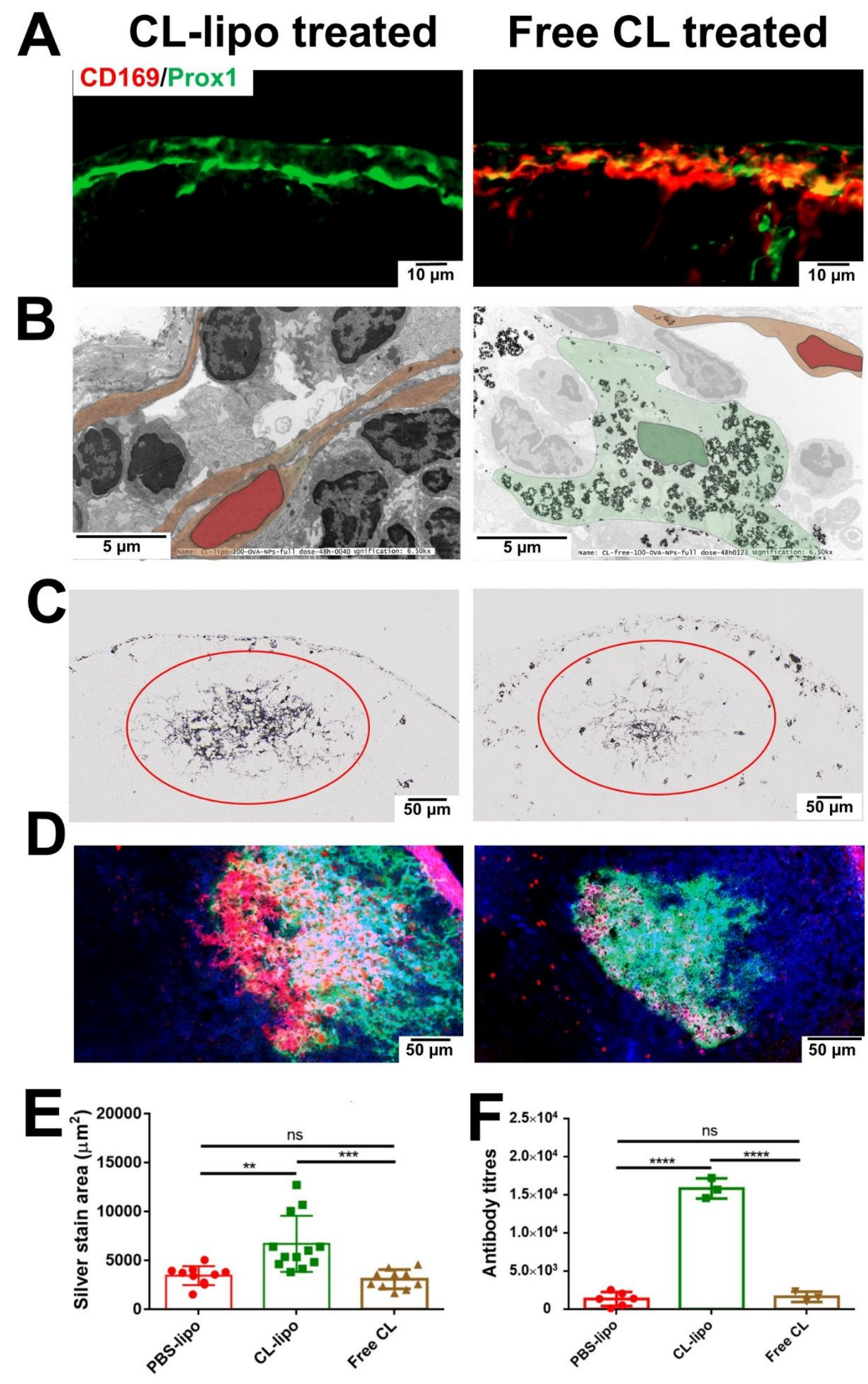
Fig. S15. Free clodronate treatment cannot efficiently remove SCS macrophage and induce robust humoral immune responses. (A) SCS macrophages remained intact in the subcapsular sinus of lymph nodes after administration of free clodronate whereas clodronate liposome treatment removed SCS macrophages. The injection doses of clodronate amount are $0.1 \mathrm{mg}$ in 20 $\mu 1$ volume. CD169 (red color) stains for SCS macrophages and Prox1 (green color) stains for LECs. (B) Representative TEM images and the enlarged images on SCS macrophages were examined after intradermal footpad injection of $100 \mathrm{~nm}$ OVA-AuNP nanovaccines after macrophage treatment using free clodronate or clodronate liposomes. The injected $100 \mathrm{~nm}$ OVA-AuNP nanovaccines were normalized based on the injected OVA antigen amount (10 $\mu \mathrm{g}) .100 \mathrm{~nm}$ OVAAuNP nanovaccines were observed in the SCS macrophages after free clodronate treatment compared to clodronate liposome treatment. Pretreatment of clodronate liposomes induced greater $100 \mathrm{~nm}$ OVA-AuNP nanovaccine accumulation in lymph node follicles and humoral immune responses compared to free clodronate (C-F). (C) Histological images of $100 \mathrm{~nm}$ OVA-AuNP nanovaccines accumulation in lymph node follicles were analyzed after 48 hours post nanovaccines intradermal footpad injection. The injected $100 \mathrm{~nm}$ OVA-AuNP nanovaccine was normalized based on the injected OVA antigen amount $(10 \mu \mathrm{g})$. Clodronate liposomes or free clodronate were administered 72 hours prior to nanovaccine injection ( $n=4$ mice/group). (E) Quantification of $100 \mathrm{~nm}$ OVA-AuNP nanovaccine accumulation in follicles at 48 hours after macrophage inhibitor administration. Assessment of (D) germinal center formation (GL7 red; CD21 green; B220 blue), and (F) antigen-specific antibody production in blood serum after administration of $100 \mathrm{~nm}$ OVA-AuNP nanovaccines at 5 weeks, Clodronate liposomes or free clodronate were administered prior to nanovaccine injection ( $\mathrm{n}=3 \mathrm{mice} / \mathrm{group}$ ). The inject 100 nm OVA-AuNP nanovaccines were normalized based on the injected OVA antigen amount (2.5 $\mu \mathrm{g})$. Data shown as mean $\pm \mathrm{SD} ; * * \mathrm{P}<0.01 ; * * * \mathrm{P}<0.001$. All $\mathrm{P}$ values are from one-way ANOVA followed by Tukey's multiple comparisons tests. 


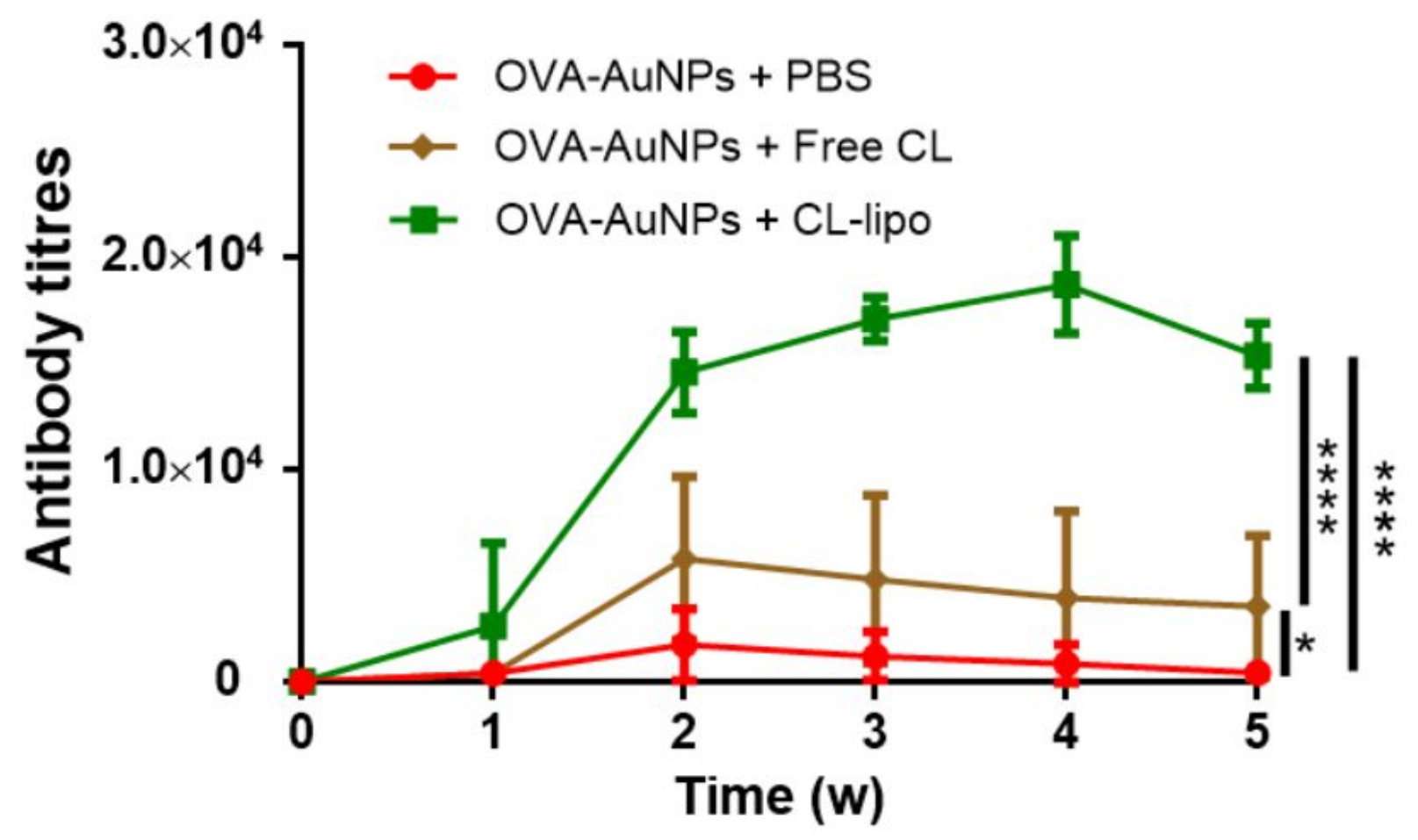

Fig. S16. Pre-mixing free clodronate with OVA-AuNPs cannot induce efficient adjuvant effects compared to clodronate liposome formulated with OVA-AuNP nanovaccines. Vaccine formulations that are composed of $100 \mathrm{~nm}$ OVA-AuNPs and free clodronate or clodronate liposomes were pre-mixed and injected through intradermal footpad into C57BL/6 mice ( $\mathrm{n}=4-7)$. Antigen OVA-specific antibody production from blood serum was measured weekly. The injected $100 \mathrm{~nm}$ OVA-AuNP nanovaccines were normalized based on the injected OVA antigen amount $(2.5 \mu \mathrm{g})$. The clodronate amount is $0.1 \mathrm{mg}$. Data shown as mean $\pm \mathrm{SD} ; * \mathrm{P}<0.05 ; * * * * \mathrm{P}<0.0001$. All P values are from two-way ANOVA followed by Tukey's multiple comparisons tests. 


\section{A}
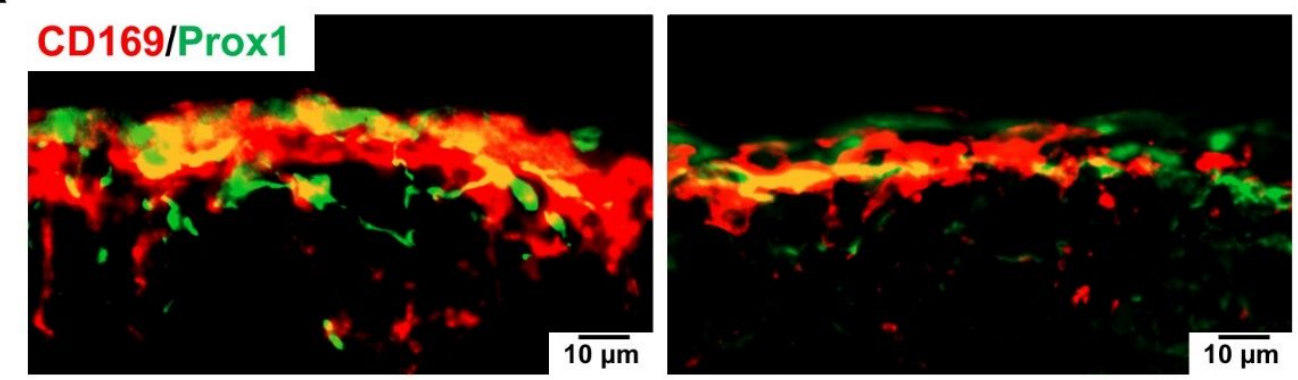

B
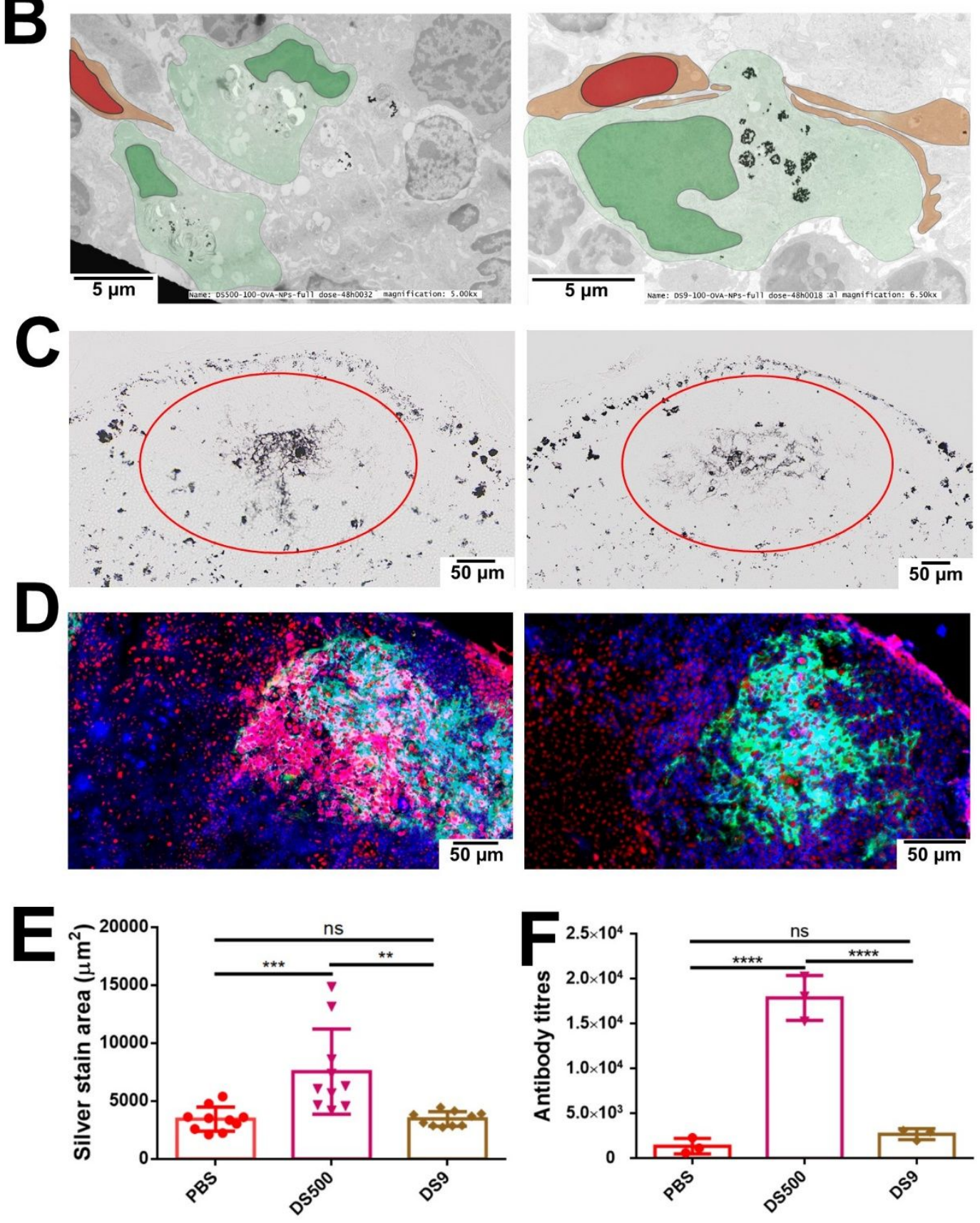
Fig. S17. Low molecular weight dextran sulfate is not sufficient to inhibit macrophage and induce robust humoral immune responses. (A) SCS macrophages remained intact in the subcapsular sinus of lymph nodes after administration of both high molecular weight DS500 (average molecular weight $>500,000 \mathrm{Da}$ ) and low molecular weight DS9 (average molecular weight 9,000 - 20,000 Da). The injection amounts are $0.1 \mathrm{mg}$ in $20 \mu \mathrm{l}$ volume. CD169 (red color) stains for SCS macrophages and Prox1 (green color) stains for LECs. (B) Representative TEM images and the enlarged images on SCS macrophages were examined after intradermal footpad injection of $100 \mathrm{~nm}$ OVA-AuNP nanovaccines after macrophage treatment using DS500 or DS9. The injected $100 \mathrm{~nm}$ OVA-AuNP nanovaccines were normalized based on the inject OVA antigen amount $(10 \mu \mathrm{g})$. Significantly less amount of 100-OVA-AuNP nanovaccines were observed in the SCS macrophages after DS500 compared to DS9 treatment. Pretreatment of DS500 induce greater $100 \mathrm{~nm}$ OVA-AuNP nanovaccine accumulation in lymph node follicles and humoral immune responses compared to DS9 (C-F). (C) Histological images of $100 \mathrm{~nm}$ OVA-AuNP nanovaccine accumulation in lymph node follicles were analyzed after 48 hours post nanovaccine intradermal footpad injection. The injected $100 \mathrm{~nm}$ OVA-AuNP nanovaccine were normalized based on the injected OVA antigen amount $(10 \mu \mathrm{g})$. DS500 or DS9 were administered 24 hours prior to nanovaccine injection ( $\mathrm{n}=4$ mice/group). (E) Quantification of $100 \mathrm{~nm}$ OVA-AuNP nanovaccine accumulation in follicles at 48 hours after macrophage inhibitor administration. Assessment of (D) germinal center formation (GL7 red; CD21 green; B220 blue), and (F) antigen-specific antibody production in blood serum after administration of $100 \mathrm{~nm}$ OVA-AuNP nanovaccine at 5 weeks, DS500 or DS9 were prior to nanovaccine injection ( $\mathrm{n}=3 \mathrm{mice} /$ group). The injected $100 \mathrm{~nm}$ OVAAuNP nanovaccine was normalized based on the inject OVA antigen amount (2.5 $\mu \mathrm{g})$. Data shown as mean $\pm \mathrm{SD} ; * \mathrm{P}<0.05 ; * * \mathrm{P}<0.01 ; * * * \mathrm{P}<0.001$. All $\mathrm{P}$ values are from one-way ANOVA followed by Tukey's multiple comparisons tests. 